\title{
Triglyceride and Glucose Index as a Screening Tool for Nonalcoholic Liver Disease in Patients with Metabolic Syndrome
}

Anca Maria Amzolini' ${ }^{1}$, Mircea-Cătălin Forțofoiu ${ }^{1 *}$, Anca Barău Al-Hija ${ }^{1}$, Ionela Mihaela Vladu ${ }^{2 *}$, Diana Clenciu ${ }^{2 *}$, Adina Mitrea ${ }^{2}$, Maria Forțofoiu ${ }^{3 *}$, Daniela Matei ${ }^{4}$, Magdalena Diaconu ${ }^{5}$, Marinela Tudor ${ }^{6}$, Elena Simona Micu ${ }^{7}$

1 Department of Internal Medicine - Medical Semiology - University of Medicine and Pharmacy of Craiova, Craiova, Romania; amzolinianca@yahoo.com, https://orcid.org/0000-0001-7664-7351 (AMA); catalin.fortofoiu@umfcv.ro, https://orcid.org/0000-0002-3341-1442 (MCF); anca_barau@yahoo.com, https://orcid.org/0000-0002-7750-8721 (ABAH);

2 Department of Diabetes, Nutrition and Metabolic Diseases - University of Medicine and Pharmacy of Craiova, Craiova, Romania; mihmitzu@yahoo.com, https://orcid.org/0000-0002-6133-4341 (IMV); dianaclenciu@yahoo.com, https://orcid.org/0000-0002-5832-0715 (DC); ada mitrea@yahoo.com, https://orcid.org/0000-0002-5310-3033 (AM)

3 Department of Emergency Medicine - University of Medicine and Pharmacy of Craiova, Craiova, Romania, maria.fortofoiu@umfcv.ro, https://orcid.org/0000-0002-2056-6779 (MF)

4 Department of Physiotherapy - University of Medicine and Pharmacy of Craiova, Craiova, Romania, mateidana30@yahoo.com, https://orcid.org/0000-0002-5681-8237 (DM)

5 Department of Pharmacology - University of Medicine and Pharmacy of Craiova, Craiova, Romania, magdalena.diaconu@umfcv.ro, https://orcid.org/0000-0003-4797-3902 (MD)

6 PhD Student - University of Medicine and Pharmacy of Craiova, Craiova, Romania; marinelasinziana@yahoo.com, https://orcid.org/0000-0001-6097-7129 (MT)

7 Clinical Municipal Hospital "Philanthropy" of Craiova, Department of Internal Medicine 2-Medical Semiology, Craiova, Romania; gvs.2005@yahoo.com, https://orcid.org/0000-0001-6153-0759 (ESM)

* Corresponding authors: catalin.fortofoiu@umfcv.ro, +4072320438 (MCF); maria.fortofoiu@umfcv.ro, +40735864289 (MF); mihmitzu@yahoo.com, +4076367081 (IMV); dianaclenciu@yahoo.com, +40745127243 (DC)

\begin{abstract}
Background: Nonalcoholic fatty liver disease (NAFLD) is regarded as a component of metabolic syndrome, which has insulin resistance (IR) as the primary physiopathological event. The aim of study was to establish the association between IR, assessed using triglyceride and glucose index (TyG), and histopathological features of NAFLD lesions. Methods: The study included patients with metabolic syndrome. Fasting plasma glucose (FPG), fasting lipid profiles and liver enzymes were measured. IR was assessed by TyG index. Liver biopsy was performed for assessment steatosis and fibrosis. Results: TyG index had a mean value of $8.93 \pm 1.45$, with a higher value in the patients with overweight $(\mathrm{p}=0.002)$ and obesity $(\mathrm{p}=0.004)$ than in the patients with normal weight. TyG index mean value of $8.78 \pm 0.65$ in subjects without NASH, $8.91 \pm 0.57$ in patients with borderline NASH and $9.13 \pm 0.55$ in patients with definite NASH. Significant difference was found between subjects without NASH and the ones with definite NASH $(\mathrm{p}=0.004)$. The analysis of the area under the ROC curve proved that TyG index is a predictor for NASH ( $p=0.043$ ). Conclusion: TyG index is a facile tool used to identify individuals at risk for NAFLD, but not for the progression of liver lesions.
\end{abstract}

Keywords: nonalcoholic liver disease, triglyceride and glucose index, metabolic syndrome, liver biopsy, obesity

\section{Introduction}

Nonalcoholic fatty liver disease (NAFLD) covers a spectrum of liver lesions (steatosis, non-alcoholic hepatosteatosis - NASH, fibrosis, cirrhosis) that appear in the absence of alcohol consumption or minimal alcohol consumption (under $20 \mathrm{~g}$ of pure alcohol for females and under $30 \mathrm{~g}$ of pure alcohol for males) in the absence of other causes of liver disease [1].

Many studies that prove the association of NAFLD with insulin resistance (IR), obesity, high blood presure and dyslipidemia regard NAFLD as a component of metabolic syndrome [2]. 
IR is considered the primary event in the physiopathology of NAFLD/NASH in many studies discussing theories related to NAFLD pathogenesis. There is a bidirectional relationship between the liver and IR: IR and secondary hyperinsulinism lead to liver disease through lipid accumulation and the liver disease exacerbates IR, transforming impaired glucose tolerance into overt diabetes mellitus through insulin metabolism impairment [3].

Liver biopsy is considered the "gold standard" for the diagnosis of liver lesions in NAFLD. Although the utility of liver biopsy, which establishes the disease subtypes, appreciates the severity and the progression of the disease, as well as certifies the liver fibrosis, it is universally recognized that this method is associated with a series of disadvantages and problems, which have led to the replacement of liver biopsy with other noninvasive procedures [4].

Even though there is an increasing interest for the study of non-invasive diagnostic methods in order to identify imaging techniques and serological markers that correlate with steatohepatitis and liver fibrosis, there is no specific biomarker that can be used for the diagnosis of NAFLD. Triglyceride accumulation and IR are the hallmarks of NAFLD, therefore triglyceride and glucose index (TyG) may predict the subsequent occurrence of NAFLD in later life. Studies showed that there is a strong and positive association between TyG and risk of NAFLD in different populations $[5,6]$.

Other studies showed that TyG index predicted NAFLD better than the homeostasis model assessment of insulin resistance (HOMA-IR), the most frequently used formula that assesses IR in clinical practice [7]. The TyG index was also positively related to the severity of liver steatosis and the presence of liver fibrosis evaluated with transient elastography in patients with NAFLD $[8,9]$.

Taking into account of these data, the aim of this study was to establish the association between IR, assessed using TyG index, and characteristic NAFLD lesions evaluated by histopathological examination.

\section{Materials and Methods}

We conducted an epidemiological, cross-sectional, non-interventional study over a period of 3 years (2017 - 2019) that included a sample of 113 subjects out of 345 patients aged over 18 years old, diagnosed with metabolic syndrome, in whom liver biopsy was performed. Based on the body mass index (BMI), the subjects were divided into 3 groups: Group 1 - 14 patients with normal weight; Group 2 - 49 patients with overweight; Group 3 - 50 patients with obesity.

The following exclusion criteria were used: pregnant or breastfeeding females, significant alcohol consumption (more than $30 \mathrm{~g} /$ day in males and more than $20 \mathrm{~g} / \mathrm{day}$ in females), patients diagnosed with hepatitis B, C \& D virus infections, patients with autoimmune liver disease, other chronic liver diseases (i.e. primary billiary cirrhosis, Wilson's disease, hereditary hemochromatosis), patients treated with hepatotoxic drugs (methotrexate, amiodarone, corticotherapy, chemotherapy, hormone therapy), as well as patients that refused the liver biopsy. Informed consent was signed, in full knowledge of the facts, by each participant in the study, after being communicated all the aspects necessary to make a decision for or against enrolling in the study.

The study was conducted in accordance with the ethical principles, stipulated in the Helsinki Declaration, in accordance with good clinical practice, respecting the right to integrity, confidentiality, the option of the subject to withdraw from the study at any time.

Data collected from the patients enrolled in the study included demographic characteristics, medical history, family history, smoking and drinking status, clinical findings and laboratory reports which were recorded accordingly in a data collection sheet. The patients' relevant physical examination was done and anthropometry data (weight and height) was collected. BMI was calculated as weight (in kilograms)/height squared (in meters). BMI was classified according to the World Health Organization (WHO) for white individuals [10] into normal weight $\left(B M I<25 \mathrm{~kg} / \mathrm{m}^{2}\right)$, overweight $\left(B M I \geq 25 \mathrm{~kg} / \mathrm{m}^{2}\right.$ and $<$ $30 \mathrm{~kg} / \mathrm{m}^{2}$ ) and obese $\left(B M I \geq 30 \mathrm{~kg} / \mathrm{m}^{2}\right)$. In all the patients, we evaluated waist 
circumference (WC) and blood pressure (BP) which was measured 3 times using an automated sphygmomanometer after the participants were relaxed and seated for more than $10 \mathrm{~min}$, the mean of the 3 determinations was recorded in the data collection sheet.

Fasting plasma glucose (FPG), fasting lipid profiles and liver enzymes were the laboratory tests that were performed in this study. After 8 to 12 hours overnight fasting, 4.0 $\mathrm{ml}$ venous blood samples were collected in a plain tube from each patient following standard procedure. The tubes were labeled with the identification numbers of the subjects. All the blood samples were kept in vertical position for 30 minutes at room temperature $\left(22^{\circ} \mathrm{C}\right.$ - $24^{\circ} \mathrm{C}$ ). Centrifugation (around $5000 \mathrm{rpm}$ ) for 5 - 10 minutes at room temperature was used to separate serum which was afterwards preserved at $-20^{\circ} \mathrm{C}$ until further analysis. The level of fasting serum triglyceride was determined by enzymatic-colorimetric method and the FPG was measured by glucose oxidase method [11]. We assessed Insulin Resistance (IR) using the triglyceride and glucose index (TyG), which was calculated by the formula [11]: TyG $=\ln$ [fasting triglycerides $(\mathrm{mg} / \mathrm{dL}) \times \mathrm{FPG}(\mathrm{mg} / \mathrm{dL}) / 2$ ]

Liver biopsy, using the Menghini technique, was performed in order to evaluate the presence of liver steatosis, NASH and fibrosis. Liver tissue fragments were fixed in 10\% formalin, then embedded in paraffin and sectioned at the microtome. The tissue slides were stained with hematoxylin-eosin, van Gieson, Gomori and Masson trichrome. Histologic grading and staging were performed according to Kleiner's and Brunts's classification (2005) presented in Table 1[12].

Table 1. Histological classification of NAFLD - modified after (12)

\begin{tabular}{|c|c|c|}
\hline Lesion & Definition & Score \\
\hline \multirow[t]{4}{*}{ Steatosis } & $<5 \%$ & 0 \\
\hline & $5-33 \%$ & 1 \\
\hline & $>33-66 \%$ & 2 \\
\hline & $>66 \%$ & 3 \\
\hline \multirow[t]{4}{*}{ Lobular inflammation } & Absent & 0 \\
\hline & 2 foci $/ 200 \times$ field & 1 \\
\hline & 2-4 foci $/ 200 \times$ field & 2 \\
\hline & $>4$ foci $/ 200 \times$ field & 3 \\
\hline \multirow[t]{3}{*}{ Hepatocyte ballooning } & Absent & 0 \\
\hline & A few balloon cells & 1 \\
\hline & Many balloon cells/ Important ballooning & 2 \\
\hline \multirow[t]{8}{*}{ Fibrosis } & Absent & 0 \\
\hline & Perisinuisoidal or periportal & 1 \\
\hline & Mild zone 3, perisinusoidal & $1 \mathrm{~A}$ \\
\hline & Moderate, zone 3, perisinusoidal & $1 B$ \\
\hline & Portal/periportal & $1 \mathrm{C}$ \\
\hline & Perisinusoidal and portal/periportal & 2 \\
\hline & Bridging fibrosis & 3 \\
\hline & Cirrhosis & 4 \\
\hline
\end{tabular}

NAFLD Activity Score (NAS) ranges from 0 to 8 and represents the sum of scores for steatosis, hepatocyte ballooning and lobular inflammation. NAS scores of 0-2 are not considered diagnostic of NASH, scores of 3-4 are considered "borderline NASH", while scores $\geq 5$ are considered "definite NASH" [13].

Demographic and clinical data were expressed as means with standard deviations and counts with percentages for continuous and discrete variables, respectively. Descriptive statistics was presented, and differences between the groups were compared using one-way ANOVA for continuous data and the chi-square test for categorical data. The 
correlations were assessed using Pearson's analysis. Multiple logistic regression analysis was used to adjust for covariates. All the statistical tests were regarded as statistically significant, with a p-value less than 0.05 (two-sided). Data were analyzed using the Statistical Package for the Social Sciences software, version 26.0 (SPSS Inc., Chicago, IL, USA).

\section{Results}

The patients enrolled in the study had a mean age of $51.57 \pm 14.14$ years old and a mean BMI of $30.55 \pm 17.17 \mathrm{~kg} / \mathrm{m}^{2}$. FPG had a mean value of $107.98 \pm 27.57 \mathrm{mg} / \mathrm{dl}$, the mean value of the triglycerides was $164 \pm 105.14 \mathrm{mg} / \mathrm{dl}$, while ALT mean value registered in the study was $44.41 \pm 39.03 \mathrm{U} / \mathrm{l}$. The characteristic of the studied patients is summarized in Table 2 . The data are presented as percentage or mean \pm standard deviation.

Table 2. Characteristics of the study patients

\begin{tabular}{|l|c|c|c|c|}
\hline \multicolumn{1}{|c|}{ Characteristics } & Normal weight & Overweight & Obesity & p \\
\hline Gender (\%) & & & & \\
Male & 14.6 & 53.7 & 31.7 & - \\
Female & 11.1 & 37.5 & 51.4 & \\
\hline Age (years) & $57.71 \pm 8.96$ & $49.20 \pm 11.60$ & $56.08 \pm 11.41$ & 0.04 \\
\hline Waist circumference (cm) & & & & \\
Male & & & & \\
Female & $101 \pm 7.34$ & $102.23 \pm 6.1$ & $115.85 \pm 6.95$ & 0.001 \\
& $82.38 \pm 1.68$ & $97 \pm 9.84$ & $110.57 \pm 9.61$ & \\
\hline FPG (mg/dl) & $88.571 \pm 14,83$ & $103.31 \pm 25.02$ & $118 \pm 50.11$ & 0.021 \\
\hline Triglycerides (mg/dl) & $123.207 \pm 56.35$ & $165.24 \pm 70.07$ & $174.50 \pm 84.72$ & 0.085 \\
\hline Hypertension (\%) & 20 & 42.9 & 54.8 & 0.317 \\
\hline ALT (U/1) & $35.2 \pm 5.79$ & $42.06 \pm 32.52$ & $49.29 \pm 28.28$ & 0.204 \\
\hline
\end{tabular}

In our study, TyG index had a mean value of $8.93 \pm 1.45$. The comparative analysis of TyG between the 3 study groups showed a higher value in the patients with overweight and obesity than in the patients with normal weight, the difference being statistical significant both when we compared subjects with normal weight and overweight $(p=0.002)$ as well as between subjects with normal weight and obesity $(p=0.004)$, but there was no statistical significance regarding the TyG value between subjects with overweight and obesity $(\mathrm{p}=0.549)$, as it is presented in Table 3 .

Table 3. The average value of TyG index in the 3 groups

\begin{tabular}{|c|c|}
\hline GROUP & TyG index \\
\hline Normal weight & $8.46 \pm 0.61$ \\
\hline Overweight & $8.93 \pm 0.55$ \\
\hline Obesity & $9.05 \pm 0.60$ \\
\hline
\end{tabular}

The degree of the liver disease (steatosis, inflammation, ballooning and fibrosis) within the 3 study groups was analyzed and the NAS was calculated accordingly.

The liver steatosis was identified in $71.43 \%$ of the patients with normal weight, in $91.84 \%$ of the patients with overweight, while all the patients with obesity presented liver steatosis (Figure 1). The statistical analysis showed a significant difference regarding the presence of liver steatosis between the patients with normal weight and overweight $(p<0.005)$, as well as between the patients with normal weight and obesity $(p<0.005)$. Furthermore, we also observed a statistically significant difference between the patients with overweight and obesity ( $\mathrm{p}=0.037)$. 


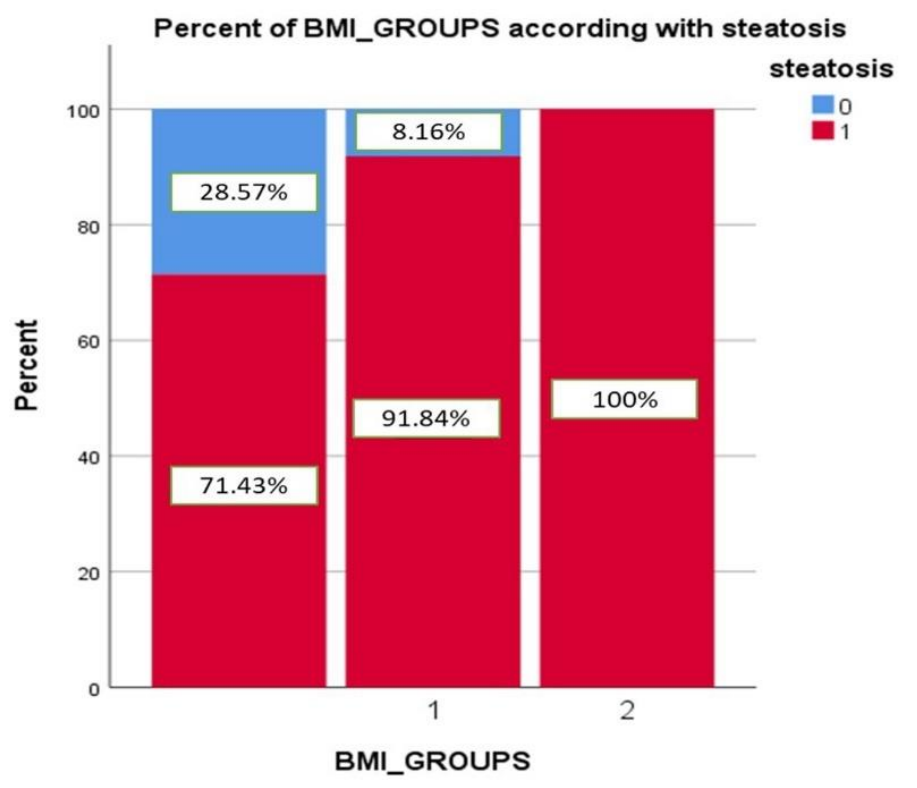

Figure 1. The distribution of liver steatosis in the study group

The liver inflammation was identified in $35.71 \%$ of the subjects with normal weight, in $57.14 \%$ of the subjects with overweight and in $60 \%$ of the patients with obesity (Figure $2)$, the difference reaching statistical significance between the subjects with normal weight and overweight $(p<0.025)$ and subjects with overweight and obesity $(p<0.01)$. There was no statistically significant difference regarding inflammation between patients with overweigh and obesity ( $p=0.773)$.

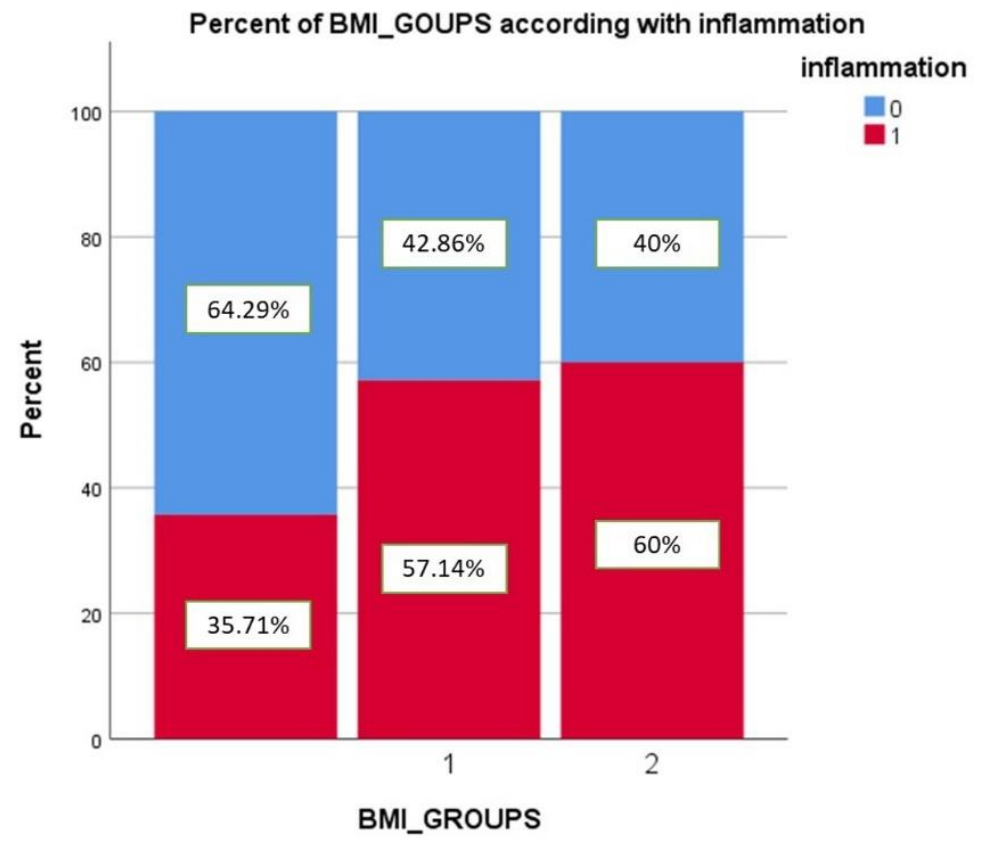

Figure 2. The distribution of liver inflammation in the study group

Ballooning was observed in $28.57 \%$ of the individuals with normal weight, in $48.98 \%$ of the patients with overweight and in $56 \%$ of the patients with obesity (Figure 3), with a statistically significant difference between the normal weight and the overweight groups $(p<0.029)$ and between normal weight and obesity groups $(p=0.003)$. There was no statistically significant difference between overweight versus obesity groups $(p=0.483)$. 


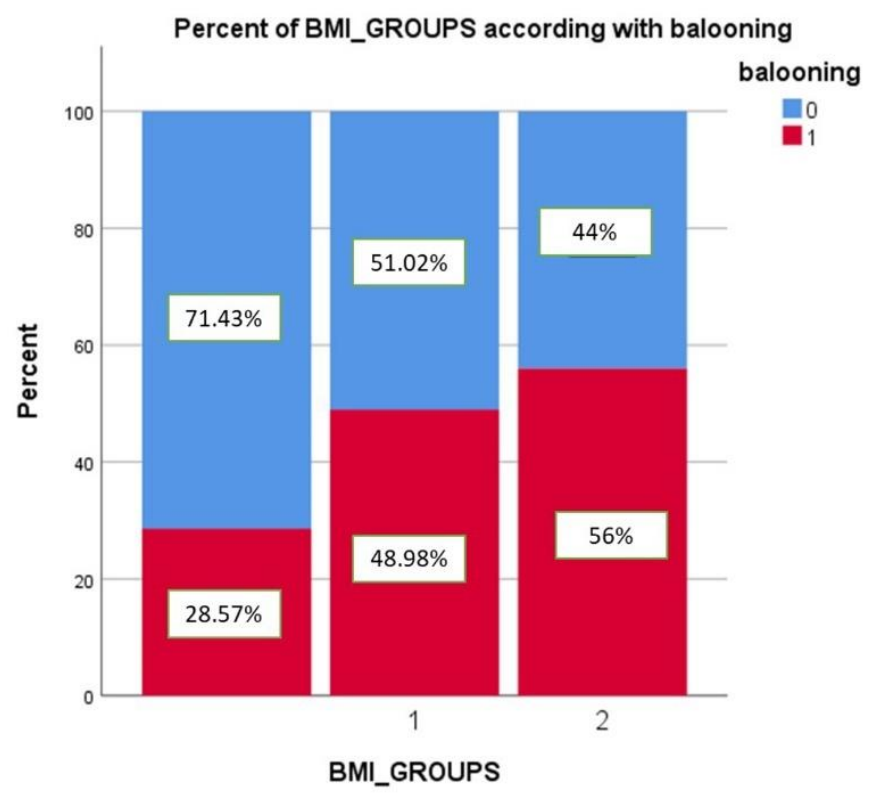

Figure 3. The distribution of hepatocyte ballooning in the study group

Fibrosis was present in $35.71 \%$ of the subjects with normal weight, in $51.02 \%$ of the patients with overweight and in $60 \%$ of the patients with obesity (Figure 4 ), reaching statistically significant difference when we compared patients with normal weight and obesity $(\mathrm{p}=0.01)$, but with no statistically significant difference between the normal weight versus overweight groups $(p=0.111)$ as well as in overweight versus obesity groups $(p=$ $0.367)$.

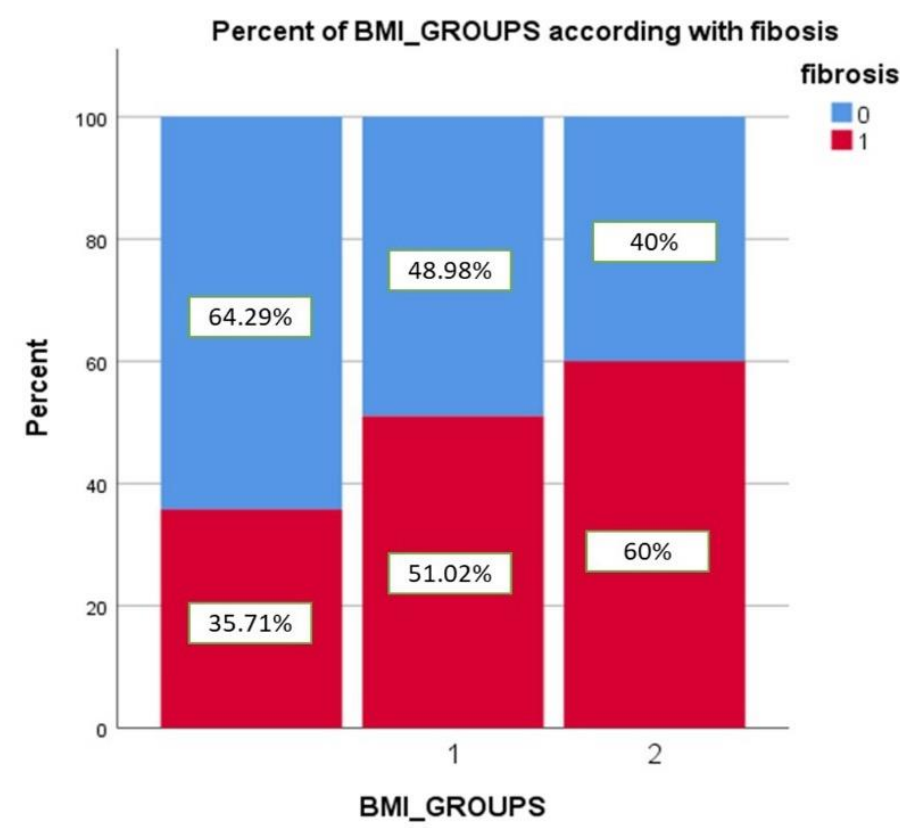

Figure 4. The distribution of liver fibrosis in the study group

NASH was calculated within the 3 study groups, dividing the patients into 3 categories: not diagnostic of NASH, borderline NASH and definite NASH. Definite NASH was reported in $14.29 \%$ of the patients with normal weight, $32.65 \%$ of the patients with overweight and in $30 \%$ of the patients with obesity. Borderline NASH was observed in $35.71 \%$ of the patients with normal weight, $32.65 \%$ in the subjects with overweight and $32 \%$ in the subjects with obesity (Figure 5). The statistical analysis showed significant differences when we compared the subjects with normal weight with both subjects with overweight 
$(\mathrm{p}<0.05)$ and obesity $(\mathrm{p}<0.05)$. There was no statistically significant difference regarding NASH categories between subjects with overweight and obesity $(p=0.717)$.

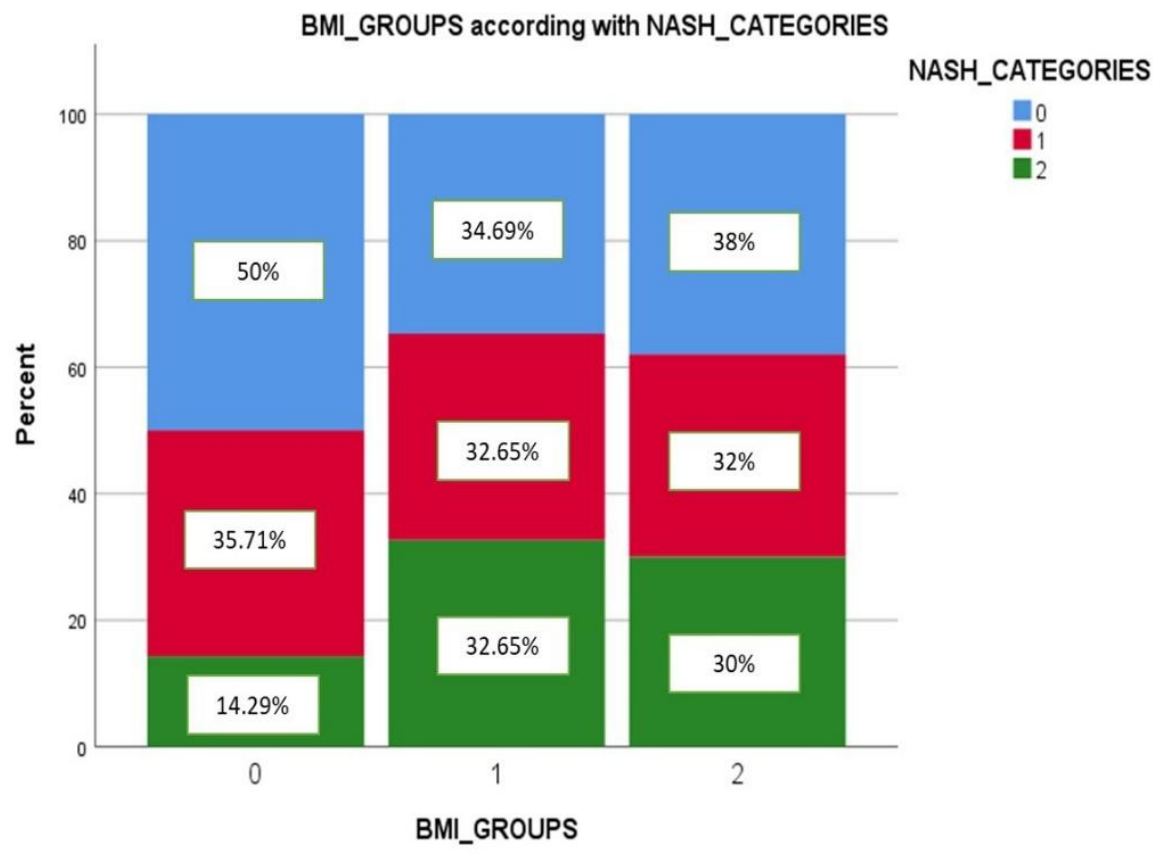

Figure 5. The distribution of the study groups according to NASH category

The TyG index was analyzed in relationship with the presence of liver steatosis, showing a mean value of $8.85 \pm 0.75$ in the subjects without liver steatosis, respectively $8.93 \pm 0.6$ in the subjects with liver steatosis, but not reaching statistical significance $(\mathrm{p}=0,708)$, as it is shown in Figure 6 .

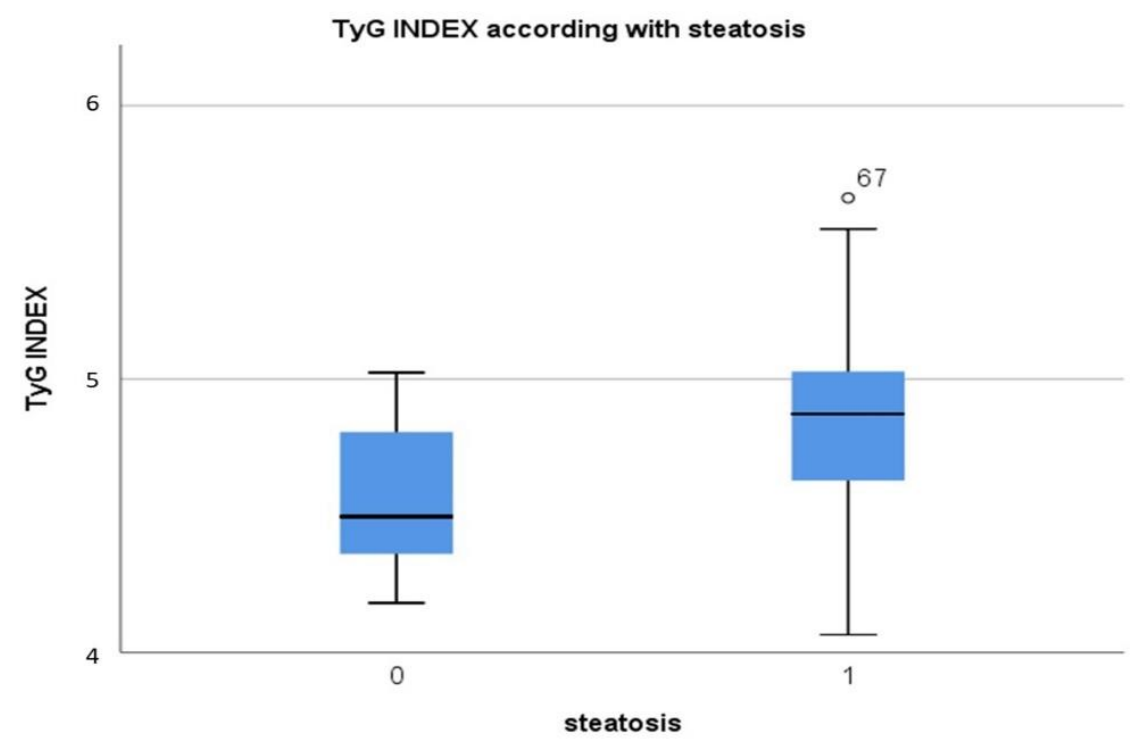

Figure 6. The study of TyG index in relationship with the presence of liver steatosis

When we studied the relationship between TyG index and liver inflammation, we identified a mean value of $8.87 \pm 0.68$ in subjects without liver inflammation, respectively $8.97 \pm 0.55$ in patients with liver inflammation, not reaching statistical significance $(\mathrm{p}=0.407)$, as presented in Figure 7 . 


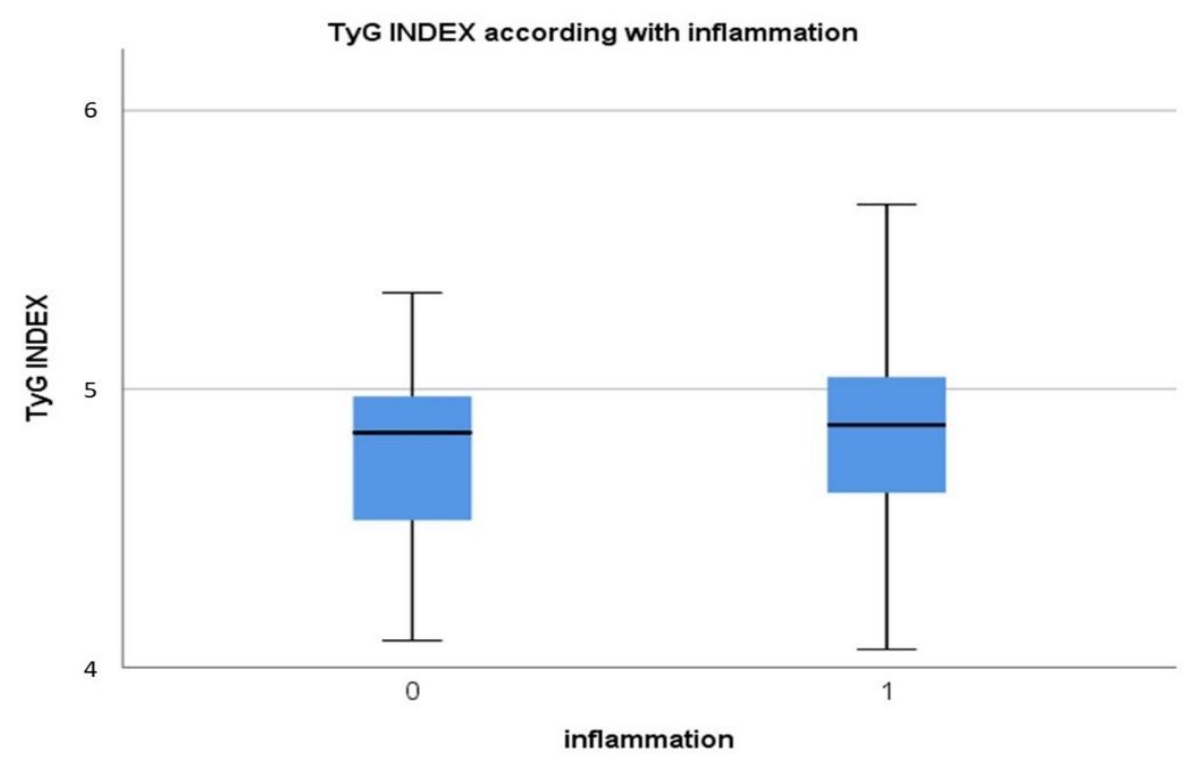

Figure 7. The study of TyG index in relationship with the presence of liver inflammation

Regarding the association between TyG index and hepatocyte ballooning, we found a mean value of $8.81 \pm 0.61$ in the subjects that did not present hepatocyte ballooning, while the subjects with hepatocyte ballooning had a mean TyG index of $9.04 \pm 0.58$, the difference being statistically significant $(\mathrm{p}=0.038)$, as it can be observed in Figure 8 .

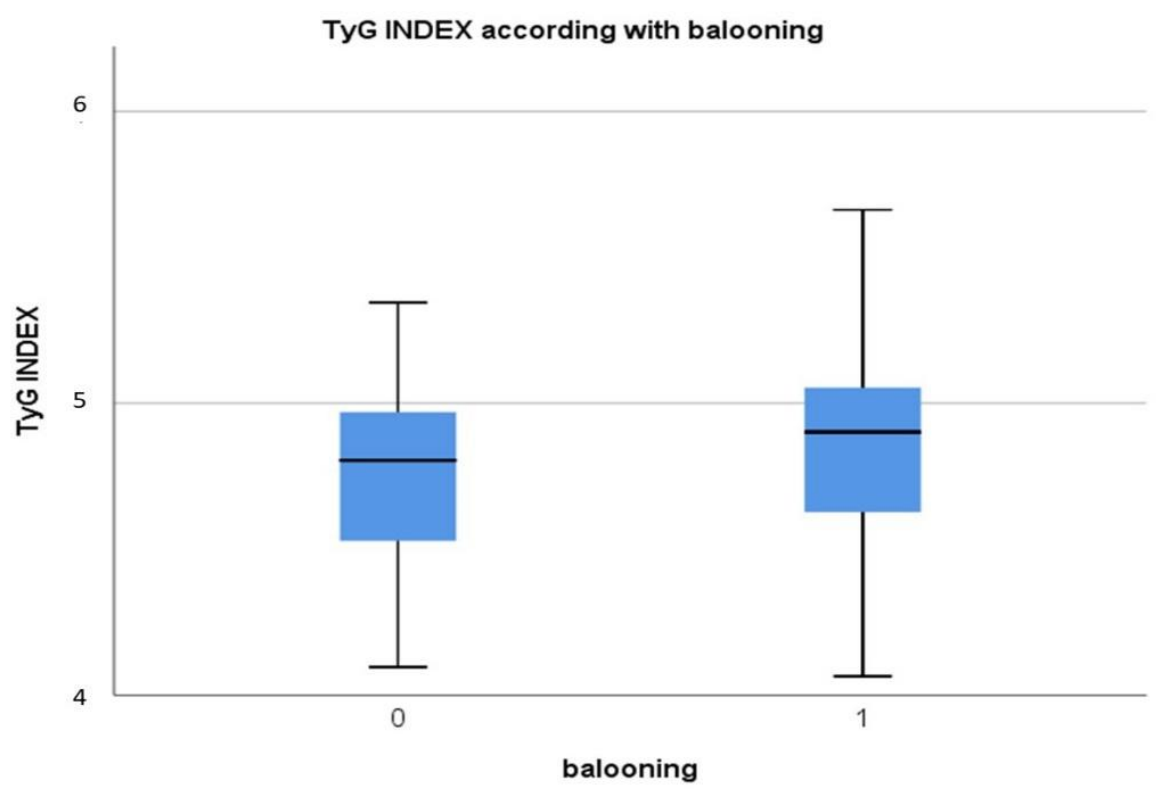

Figure 8. The study of TyG index in relationship with the presence of hepatocyte ballooning

Figure 9 presents the study of the TyG index in patients with liver fibrosis. TyG had a mean value of $9.01 \pm 0.58$ in the patients presenting liver fibrosis, while the subjects without liver fibrosis had a mean TyG value of $8.82 \pm 0.62$, the difference not reaching statistical significance $(\mathrm{p}=0.092)$. 


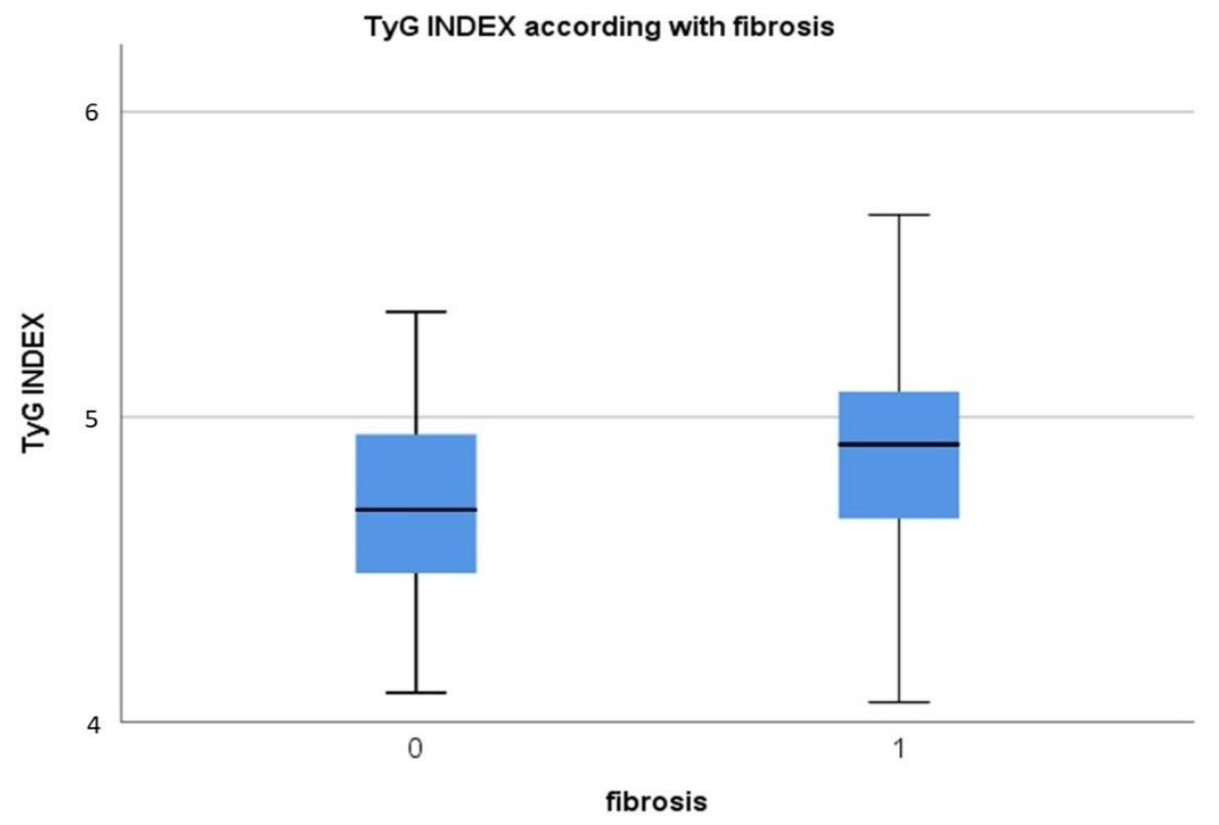

Figure 9. The study of TyG index in relationship with the presence of liver fibrosis

The associations between TyG index and NASH categories were also studied. We found a mean TyG index value of $8.78 \pm 0.65$ in subjects without NASH, $8.91 \pm 0.57$ in patients with borderline NASH and $9.13 \pm 0.55$ in patients with definite NASH, but the only statistically significant difference was found between subjects without NASH and the ones with definite NASH ( $\mathrm{p}=0.004)$, as presented in Figure 10.

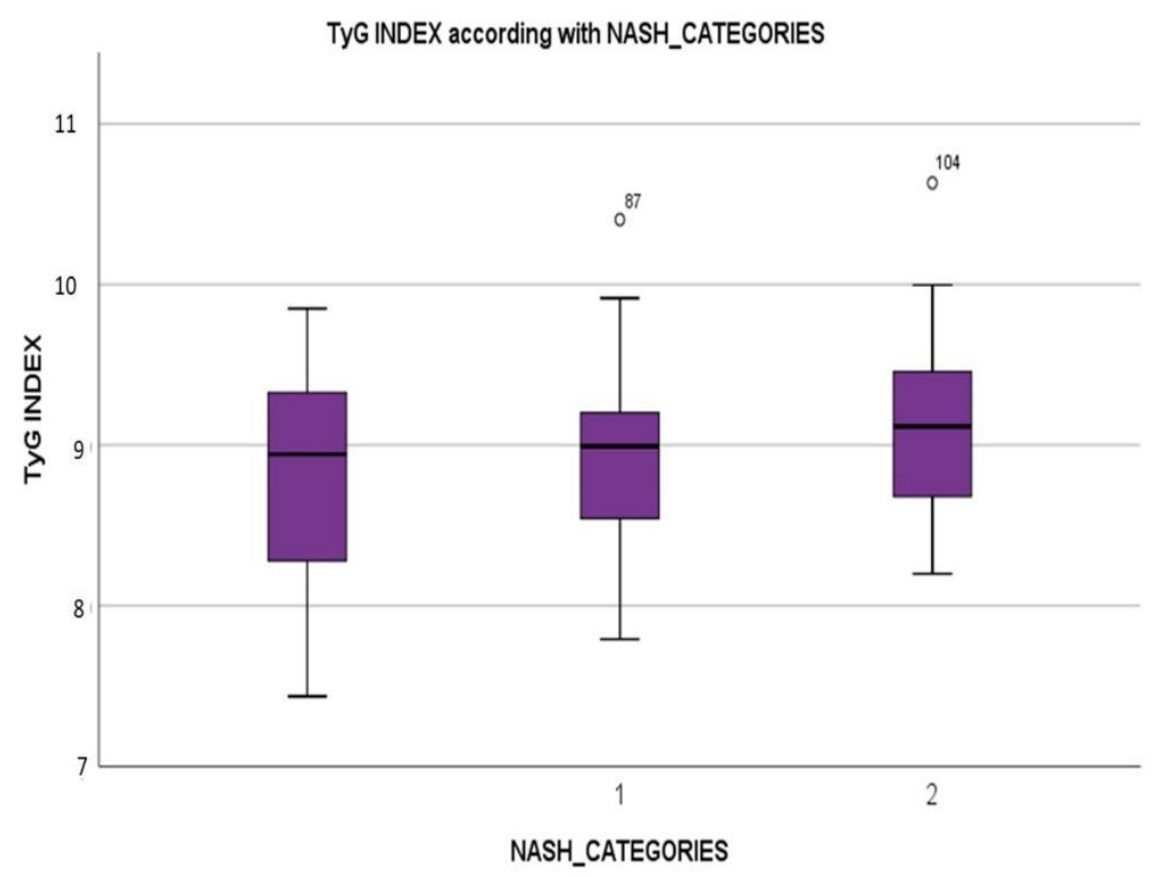

Figure 10. The study of TyG index in relationship with NASH categories

The analysis of the area under the ROC curve showed a value of 0.635 (Figure 11), proving that TyG index is an independent predictor for NASH $(\mathrm{p}=0.043)$, however this value is not high enough to be used in order to determine a cut-off value of TyG index for the presence of NASH. 


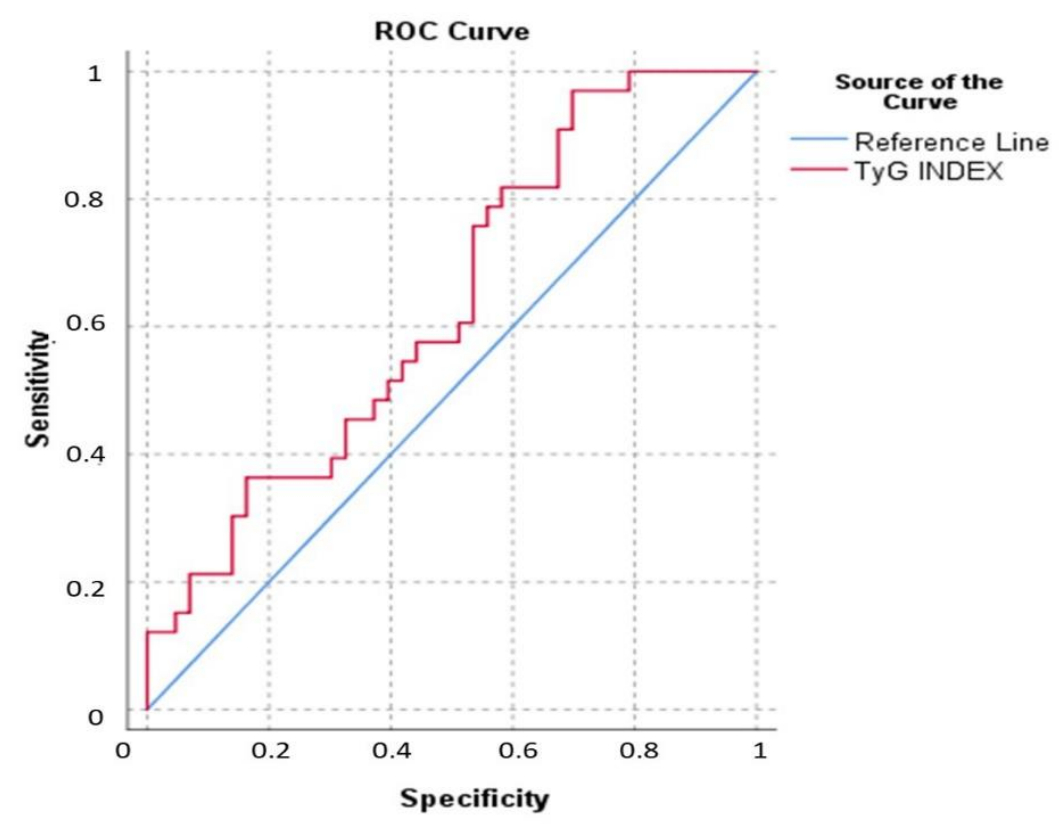

Figure 11. The area under the ROC curve for TyG index as a predictor of NASH

\section{Discussion}

NAFLD is considered a major public health problem worldwide. Obesity, type 2 diabetes mellitus and dyslipidemia, in the absence of alcohol consumption, are the most important risk factors for NAFLD, having IR as the main pathogenic mechanism.

In the medical literature, the prevalence of liver steatosis in the subjects with obesity is estimated at $73-92 \%$, reaching $100 \%$ in subjects with diabetes and morbid obesity [14]. In our study, steatosis had a frequency of $92.03 \%$, being identified in $71.43 \%$ of the study subjects with normal weight, in $91.84 \%$ of the subjects with overweight and in all the subjects with obesity $(100 \%)$. Our results, which show a high percentage of liver steatosis in the patients with normal weight, can be explained by the inclusion criteria used in this study, which enrolled only subjects diagnosed with metabolic syndrome. All the subjects that presented normal weight had an abdominal circumference above the normal value [15], suggestive for visceral obesity, which implies triglycerides accumulation in the liver [16]. Furthermore, abdominal obesity is considered a risk factor for NAFLD, even in subjects with BMI within the normal range [17].

Regarding NASH, the prevalence of this disorder is estimated to $2-7 \%$ of the general population, and $34-40 \%$ in patients with abnormal liver enzymes but in the absence of viral hepatitis markers [16], reaching $37 \%$ in patients with morbid obesity [18]. According to data published till the present, in $12-40 \%$ of the subjects, liver steatosis evolves into steatohepatitis and incipient fibrosis within 8 to 13 years [19]. In our study, NASH had a frequency of $32.69 \%$ in the subjects with liver steatosis, while liver fibrosis had a frequency of $58.65 \%$.

TyG index, which uses regular blood tests (FPG, serum triglycerides), is a non-invasive marker of IR as well as a good predictor for NAFLD $[20,21]$. Studies showed an association of liver steatosis with TyG values above 8.5-8.85 [5,6,22,24]. The mean TyG value reported in our study is $8.93 \pm 1.45$, with no statistic significant difference between subjects that presented liver steatosis and the ones that did not present this type of liver disorder. Although we found a small percentage of subjects that did not present liver steatosis, the mean value of TyG in these patients was $8.85 \pm 0.75$, higher than the cut-off presented in previous studies. However, when interpreting the results of our study, it must be taken 
into consideration the fact that all the patients enrolled in the study were diagnosed with metabolic syndrome, disorder that is associated with IR and an increased TyG index. Furthermore, the lack of correlation between our results and the data reported in the medical literature, associating TyG with both the presence and the severity of liver steatosis [22], can be explained by different diagnostic method, as most of the published studies assessed liver steatosis using non-invasive methods, while the diagnosis of liver steatosis in our study was established after liver biopsy and histopathological examination.

Regarding TyG index as a marker of NASH, in this study we found statistically significant differences $(\mathrm{p}=0.004)$ between TyG index value in patients without NASH and definite NASH according to NAS, calculated using histopathological criteria. The analysis of the area under the ROC curve showed a value of $0.635(\mathrm{p}=0.043)$, however this value was not high enough in order to establish a cut-off value that can be used a marker of NASH. Further studies, including a higher number of patients are needed in order to evidence a cut-off value that could be used in the clinical practice.

In our study, liver fibrosis was met in $53.09 \%$ of the studied patients. The frequency of liver fibrosis in patients with normal weight, but with abdominal obesity, according to waist circumference, was $37 \%$; however, the degree of fibrosis in these patients was not higher than F1. The TyG index mean values varied between $8.82 \pm 0.62$ in patients without liver fibrosis and $9.01 \pm 0.58$ in patients with liver fibrosis, regardless of the degree of fibrosis, the difference not reaching statistical significance. The studies reporting till present a correlation between TyG and liver fibrosis have assessed this parameter through noninvasive methods $[23,24,25]$, such as scoring systems or transient elastography - fibroscan.

\section{Conclusions}

Our study showed a correlation between IR assessed using TyG index and histopathological components of NAFLD, especially NASH, however, without being able to identify a TyG cut-off value suggestive for steatohepatitis. TyG index can be considered a facile tool used to identify individuals at risk for NAFLD, but not for the progression of liver lesions in order to select the patients in whom early prevention measures could prevent the evolution of liver steatosis.

Author Contributions: Conceptualization, AMA, ESM, MF, and MCF.; methodology, DC, IMV, AM, AMA.; software, MCF, MF, MT, ESM.; validation, ABAH, ESM. and MF.; formal analysis, MD, DM, MT.; investigation, MD, DM, MT, IVM, DC.; resources, IMV, AM, DC, AMA, ESM, ABAH.; data curation, IMV, AM, DC, MCF.; writing - original draft preparation, AMA, ABAH, DM, MD.; writing - review and editing, DM, AM, DC, IMV, ESM; visualization MCF, MF, MT, MD; supervision, MCF, IMV, AM, DC; project administration, DC, MD, AM, MF, AMA. All authors have read and agreed to the published version of the manuscript. The authors contributed equally to the manuscript and thus share first authorship.

Funding: This research received no external funding.

Institutional Review Board Statement: The study was conducted according to the guidelines of the Declaration of Helsinki in accordance with good clinical practice, respecting the right to integrity, confidentiality, the option of the subject to withdraw from the study at any time. Ethical review committee: Ethics Commission of the Municipal Clinical Hospital Philanthropy from Craiova. Approval number: 18086/07.11.2017

Informed Consent Statement: Informed consent was obtained from all subjects involved in the study.

Acknowledgments: In this section, you can acknowledge any support given which is not covered by the author contribution or funding sections. This may include administrative and technical support, or donations in kind (e.g., materials used for experiments).

Conflicts of Interest: The authors declare no conflict of interest.

\section{References}

1. Alba LM and Lindor K: Review article: nonalcoholic fatty liver disease. Aliment Pharmacol Ther 17: 977-986, 2003

2. de Alwis NMW and Day CP: Non-alcoholic fatty liver disease: The mist gradually clears. J Hepatol 48: S104-112, 2008 
3. Bugianesi E, McCullough A and Marchesini G: Insulin Resistance: A Metabolic Pathway to Chronic Liver Disease. Hepatology 42: 987-1000, 2005

4. Neagoe CD, Avramescu ET, Amzolini AM, Singer CE, Ianosi SL, Genunche AV, Ianosi GN, Farmazon AS, Cazacu SM and Popescu M: The accuracy of NAFLD diagnosis using mathematical scores in active young obese patients. Journal of Sport and Kinetic Movement. No 34,Vol. II/2019

5. Zhang SJ, Du TT, Zhang JH, Lu HM, Lin X, Xie JH, Yang Y and Yu XF: The Triglyceride and Glucose Index (TyG) Is an Effective Biomarker to Identify Nonalcoholic Fatty Liver Disease. Lipids Health Dis 16(1): 15, 2017

6. Zheng RJ, Du ZN, Wang MM, Mao YS and Mao WJ: A Longitudinal Epidemiological Study on the Triglyceride and Glucose Index and the Incident Nonalcoholic Fatty Liver Disease. Lipids Health Dis 17(1): 262, 2018

7. Lee SB, Kim MK, Kang S, Park K, Kim JH, Baik SJ, Nam JS, Ahn CW and Park JS: Triglyceride glucose index is superior to the homeostasis model assessment of insulin resistance for predicting nonalcoholic fatty liver disease in Korean adults. Endocrinol Metab (Seoul) 34(2): 179-86, 2019

8. Inavolu P, Singla N, Nunsavata K and Bhashyakarla RK: 14. Triglyceride and Glucose Index (TYG) Index as an Screening Biomarker to Identify Nonalcoholic Fatty Liver Disease. Journal of Clinical and Experimental Hepatology 8: S41, 2018

9. Kim MK, Kim JH, Park K, Lee SB, Nam JS, Kang S, Park JS, Ahn CW and Kim YS: Relationship between the Triglyceride Glucose Index and the Presence and Fibrosis of Nonalcoholic Fatty Liver Disease in Korean Adults. Diabetes 67: 612, 2018

10. Weir CB and Jan A: BMI Classification Percentile And Cut Off Points. In: StatPearls [Internet]. Treasure Island (FL): StatPearls Publishing (ed), 2021

11. Simental-Mendía LE, Rodríguez-Morán M and Guerrero-Romero F: The Product of Fasting Glucose and Triglycerides as Surrogate for Identifying Insulin Resistance in Apparently Healthy Subjects. Metab Syndr Relat Disord 6(4): 299-304, 2008

12. Kleiner DE, Brunt EM, Van Natta M, Behling C, Contos MJ, Cummings OW, Ferrell LD, Liu YC, Torbenson MS, Unalp-Arida A, et al: Design and validation of a histological scoring system for non-alcoholic fatty liver disease. Hepatology 41: 1313-1321, 2005

13. Brunt EM, Kleiner DE, Wilson LA, Belt P, Neuschwander-Tetri BA; NASH Clinical Research Network (CRN): Nonalcoholic fatty liver disease (NAFLD) activity score and the histopathologic diagnosis in NAFLD: distinct clinicopathologic meanings. Hepatology 53(3): 810-820, 2011

14. Erickson S: Nonalcoholic fatty liver disease. J Lipid Res 50: S412-416, 2009

15. Alberti KG, Eckel RH, Grundy SM, Zimmet PZ, Cleeman JI, Donato KA, Fruchart JC, James WP, Loria CM, Smith SC Jr, et al: Harmonizing the Metabolic Syndrome A Joint Interim Statement of the International Diabetes Federation Task Force on Epidemiology and Prevention; National Heart, Lung, and Blood Institute; American Heart Association; World Heart Federation; International Atherosclerosis Society; and International Association for the Study of Obesity. Circulation 120: 16401645,2009

16. Tarantino G, Saldalamacchia G, Conca P and Arena A: Non-alcoholic Fatty Liver Disease: Further Expression of the Metabolic Syndrome. J Gastroenterol Hepatol 22(3): 293-303, 2007

17. Oka R, Miura K, Sakurai M, Nakamura K, Yagi K, Miyamoto S, Moriuchi T, Mabuchi H, Yamagishi M, Takeda Y, et al: Comparison of waist circumference with body mass index for predicting abdominal adipose tissue. Diabetes Res Clin Pract 83: 100-105, 2009

18. Dowman JK, Tomlinson JW and Newsome PN: Systematic Review: The Diagnosis and Staging of Non-alcoholic Fatty Liver Disese and Non-alcoholic Steatohepatitis. Aliment Pharmacol Ther 33(5): 525-540, 2011

19. Ekstedt M, Franzén LE, Mathiesen UL, Thorelius L, Holmqvist M, Bodemar G, Kechagias S. Long-term follow-up of patients with NAFLD and elevated liver enzymes. Hepatology 44: 865-873, 2006

20. Guerrero-Romero F, Simental-Mendía LE, González-Ortiz M, Martínez-Abundis E, Ramos-Zavala MG, Hernández-González SO, Jacques-Camarena $\mathrm{O}$ and Rodríguez-Morán M: The product of triglycerides and glucose, a simple measure of insulin sensitivity. Comparison with the euglycemic-hyperinsulinemic clamp. J Clin Endocrinol Metab 95: 3347-3351, 2010

21. Simental-Mendia LE, Simental-Mendia E, Rodriguez-Hernandez H, Rodriguez-Moran M and Guerrero-Romero F. The product of triglycerides and glucose as biomarker for screening simple steatosis and NASH in asymptomatic women. Ann Hepatol 15: 715-720, 2016

22. Hossain S, Sultana S, Zaman K, Shafiq S, Rahman A, Hossain S, Islam S, Uddin M, Faroque M, Ferdoushi S, et al: Triglyceride and Glucose Index (TyG) Is a Reliable Biomarker to Predict Non-Alcoho- lic Fatty Liver Disease. Journal of Biosciences and Medicines 8: 124-136, 2020

23. Guo W, Lu J, Qin P, Li X, Zhu W, Wu J, Xu N and Zhang Q. The triglyceride-glucose index is associated with the severity of hepatic steatosis and the presence of liver fibrosis in non-alcoholic fatty liver disease: a cross-sectional study in Chinese adults. Lipids Health Dis 19(1): 218, 2020

24. Petrescu Ileana Octavia, Biciusca V., Taisescu, C.I., Alexandru D.O., Taisescu Oana, Comanescu Maria Victoria, Petrescu Fl., Popescu IAS, Trasca Diana-Maria, Fortofoiu M.C., Silosi, C.A., Fortofoiu Maria. Histological factors that predict the liver fibrosis in patients with chronic hepatitis C. Romanian Journal of Morphology and Embryology 57(2) Suppl:759-765, 2016

25. Fortofoiu Maria, Fortofoiu M.C. Clinical, histological, immunohistochemical and morphometric aspects in patients with chronic hepatitis of alcoholic and viral etiology. Nano, bio- and green technologies for a sustainable future Conference Proceedings, SGEM 2016, Vol. I, pp. 471-478 